Pacific

Journal of

Mathematics

\title{
A CAPILLARITY PROBLEM FOR COMPRESSIBLE LIQUIDS
}

Maria Athanassenas AND JUlie Clutterbuck 


\title{
A CAPILLARITY PROBLEM FOR COMPRESSIBLE LIQUIDS
}

\author{
Maria Athanassenas and Julie Clutterbuck
}

\begin{abstract}
We study the existence and regularity of solutions to the capillarity problem for compressible liquids in a tube. We introduce an appropriate space of functions of bounded variation, in which the energy functional introduced recently by Robert Finn can be defined. We prove existence of a locally Lipschitz minimizer in this class.
\end{abstract}

\section{Introduction}

A capillary surface is the free interface between two immiscible fluids (liquid-gas or liquid-liquid); as an authoritative introduction we refer to [Finn 1986]. Extensive work has been published on the behavior of such interfaces when one liquid is assumed to be incompressible. Finn [2001; 2004] introduced a new model, in which the assumption of incompressibility was removed.

The established techniques used for finding existence and regularity for the incompressible model may be divided into two groups: classical PDE techniques for surfaces of prescribed mean curvature, as, for example, in [Ladyzhenskaya and Ural'tseva 1970; Huisken 1985; Gilbarg and Trudinger 2001, Chapter 16]; and functions of bounded variation and sets of finite perimeter setting for minimizing the energy, as, for example, in [Emmer 1973; Gerhardt 1974; Giusti 1980; Gonzalez et al. 1980; Huisken 1985]. In contrast, results concerned with compressible liquids are very recent and comparatively few [Athanassenas and Finn 2006; Finn and Luli 2007].

Here, we consider a capillary tube of cross section $\Omega \subset \mathbb{R}^{n}$, which is bounded, simply connected and has Lipschitz boundary $\Sigma:=\partial \Omega$. We will also assume that it satisfies an interior sphere condition of radius $R$.

The capillary surface $\mathscr{Y}$ is given as the graph of a function $u$ over the domain $\Omega$. We assume uniform downwards gravity $g$ and consider a compressible fluid of density $\Phi$. (In the incompressible case, $\Phi$ is constant.)

One can assume prescribed mass $M$, but the results in the present paper are for an infinite container.

MSC2000: 49Q20, 76B45, 76N10.

Keywords: capillarity, function of bounded variation, compressible liquid. 
We consider the energy for a capillary surface to consist of the following components:

Energy of the free surface (surface tension):

$$
E_{\mathscr{S}}=\frac{\sigma}{\rho_{0}} \int_{\Omega} \Phi\left(u ; p_{0}\right) \sqrt{1+|D u|^{2}} d x ;
$$

Potential energy:

$$
W=g \int_{\Omega} \int_{0}^{u} h \Phi\left(h ; p_{0}\right) d h d x
$$

Wetting energy:

$$
E_{\Sigma}=-\sigma \int_{\Sigma} \beta \int_{0}^{u} \Phi\left(h ; p_{0}\right) d h d s .
$$

Here $\beta \in L^{\infty}(\Sigma)$ is the relative adhesion coefficient, satisfying $|\beta| \leq 1-a$ with $a>0 ; \sigma$ and $g$ are the surface tension and gravitational constants; $\Phi\left(h ; p_{0}\right)$ is the density function depending on height $h$ and pressure $p$, which we assume to be given by one of the two models proposed in [Finn 2001; Athanassenas and Finn 2006]. In the following, $p_{0}$ and $\rho_{0}$ will denote pressure and density at a reference level $u \equiv 0$.

Mass: In the case of a mass constraint, a term $\lambda M$ is added to the energy, where $\lambda$ is a Lagrange multiplier and the mass is

$$
M=\int_{\Omega} \int_{0}^{u} \Phi\left(h ; p_{0}\right) d h d x .
$$

The total energy (and in particular the wetting energy) need not be positive.

A smooth minimizer of the total energy $E_{\varphi}+W+E_{\Sigma}+\lambda M$ will satisfy the Euler-Lagrange equation

$$
\operatorname{div} \frac{D u}{\sqrt{1+|D u|^{2}}}=\frac{g \rho_{0}}{\sigma} u+\frac{D_{1} \Phi\left(u ; p_{0}\right)}{\Phi\left(u ; p_{0}\right)} \frac{1}{\sqrt{1+|D u|^{2}}}+\lambda \frac{\rho_{0}}{\sigma} \text { on } \Omega,
$$

with boundary condition

$$
\beta=\frac{1}{\rho_{0}} \frac{D u \cdot v}{\sqrt{1+|D u|^{2}}} \text { on } \Sigma,
$$

via standard calculus of variations techniques.

The present paper is based on one of the models proposed by Finn for an isothermal fluid: the density is assumed to be linear in the pressure, from which one obtains that $\Phi\left(h ; p_{0}\right)=\rho_{0} e^{-\chi g h}$, for some positive constant $\chi$.

We may assume that $\chi=1, g=1, \rho_{0}=1, \sigma=1$; other values of these constants correspond to different weightings on the components of the energy (that is, our energy becomes $\gamma_{1} E_{\varphi}+\gamma_{2} W+\gamma_{3} E_{\Sigma}+\gamma_{4} \lambda M$ for $\gamma_{i}>0$ ), and a scaling of the domain $\Omega$. 
With these conventions

$$
\Phi\left(h ; p_{0}\right)=e^{-h},
$$

and the diverse components of the energy are as follows:

$$
\begin{aligned}
E_{\mathscr{S}} & =\int_{\Omega} e^{-u} \sqrt{1+|D u|^{2}} d x, \\
W & =\int_{\Omega} \int_{0}^{u} h e^{-h} d h d x=\int_{\Omega}\left(1-e^{-u}(1+u)\right) d x, \\
E_{\Sigma} & =-\int_{\Sigma} \beta \int_{0}^{u} e^{-h} d h d s=-\int_{\Sigma} \beta\left(1-e^{-u}\right) d s, \\
M & =\int_{\Omega} \int_{0}^{u} e^{-h} d h d x=\int_{\Omega}\left(1-e^{-u}\right) d x .
\end{aligned}
$$

As we are dealing with the case of an infinite container, we choose $\lambda=0$. Without loss of generality (this will be shown when necessary, in Lemma 3.6) we may set $\gamma_{i} \equiv 1$, and then seek to minimize the energy

$$
\mathscr{g}(u):=E_{\mathscr{S}}(u)+W(u)+E_{\Sigma}(u) .
$$

The following results have been recently obtained for the capillarity problem of a compressible fluid.

For slightly compressible fluids, Finn [2001] introduced the model we are using here. In the case of a tube closed at the bottom, he found that the necessary condition on the mass for existence of a solution is $M<\rho_{0}|\Omega| / \chi g$.

The boundary contact angle is denoted by $\gamma$, with $\cos \gamma=\beta$. For a circular tube, Finn and Luli [2007] showed that when $0 \leq \gamma<\pi$ there is at least one symmetric solution of the problem, and that the height of this solution will lie above any prescribed level if $M$ is sufficiently large. If $\gamma \leq \pi / 2$, the solution is unique among symmetric solutions with that mass.

The classical PDE approach was chosen by Athanassenas and Finn [2006] to study the unconstrained case, including the situation where the term $\left(\rho_{0} g / \sigma\right) u$ on the right-hand side of the prescribed mean curvature equation (1-1) is replaced by $\left(e^{\chi g u}-1\right)\left(\rho_{0}-\chi p_{0}\right) /(\sigma \chi)$. The results vary depending on the regularity of the boundary of the domain: for smooth domains height and gradient estimates and existence of smooth solutions were obtained, but only variational solutions for domains with Lipschitz boundaries. As with the incompressible case, they observe that existence of solutions depends discontinuously on the opening angle of the corners of the domain. In the case with the alternative right-hand side of (1-1), they show nonexistence whenever the domain is small, that is, when $|\Omega|\left(\rho_{0}-\chi p_{0}\right) /(\sigma \chi)<-|\Sigma| \beta$ (here $\beta$ is taken to be constant).

In the present paper we use techniques involving functions of bounded variation. 
In Section 2, we introduce BV, the space of functions of bounded variation. After a transformation of $u$, the weighted surface area term is well defined in BV. Transforming the remaining components of the energy gives us a new energy, $\mathscr{F}_{1}$. We collect and give simple proofs of known results for the reader's convenience.

In Section 3, we prove height estimates. In order to do so, we use two (possibly new) extensions to the well-known Stampacchia lemma.

In Section 4, we show that the energy functional is bounded from below and that a minimizing sequence for the energy functional is uniformly bounded in the $\mathrm{BV}$-norm. Existence then follows via the standard compactness theorem and by the lower semicontinuity of the functional.

Finally, in Section 5 we show that there exists a locally Lipschitz minimizer, which is analytic in $\Omega$ and has bounded gradient up to the boundary.

\section{The energy in the isothermal case}

Let $\Omega$ be open, simply connected and have a Lipschitz boundary. As in [Giusti 1984; Bemelmans and Dierkes 1987], we define

$$
\begin{gathered}
\int_{\Omega} \sqrt{1+|D u|^{2}} \\
\quad:=\sup \left\{\int_{\Omega} g_{n+1}+u \operatorname{div}_{n} g d x: g_{i} \in C_{0}^{1}(\Omega) \forall i=1, \ldots, n+1, \sum_{i=1}^{n+1} g_{i}^{2} \leq 1\right\}, \\
\int_{\Omega}|D u|:=\sup \left\{\int_{\Omega} u \operatorname{div}_{n} g d x: g_{i} \in C_{0}^{1}(\Omega) \forall i=1, \ldots, n, \sum_{i=1}^{n} g_{i}^{2} \leq 1\right\},
\end{gathered}
$$

where $\operatorname{div}_{n}$ is the divergence of the first $n$ components: $\operatorname{div}_{n} g=\sum_{i=1}^{n} D_{i} g_{i}$.

One then defines the spaces

$$
\begin{aligned}
\operatorname{BV}(\Omega) & :=\left\{u \in L^{1}(\Omega): \int_{\Omega} \sqrt{1+|D u|^{2}}<\infty\right\}, \\
\mathrm{BV}^{+}(\Omega) & :=\{u \in \operatorname{BV}(\Omega): u \geq 0 \text { almost everywhere in } \Omega\} .
\end{aligned}
$$

When $|\Omega|<\infty$, this definition of $\operatorname{BV}(\Omega)$ is equivalent to the classic one given in [Giusti 1984].

In the case that $u \in C^{1}(\Omega)$, the surface energy term $E_{\mathscr{S}}=\int_{\Omega} e^{-u} \sqrt{1+|D u|^{2}} d x$ may be simplified by writing $v=e^{-u}$, so $E_{\varphi}=\int_{\Omega} \sqrt{v^{2}+|D v|^{2}} d x$, which bears a close resemblance to the integral $\int_{\Omega} \sqrt{v+|D v|^{2} / 4} d x$ investigated in [Bemelmans and Dierkes 1987]; see also [Dierkes and Huisken 1990].

The focus of our investigation now shifts to $v$, rather than $u$ itself. Define

$$
\begin{aligned}
& \int_{\Omega} \sqrt{v^{2}+|D v|^{2}} \\
& \quad:=\sup \left\{\int_{\Omega} v\left(g_{n+1}+\operatorname{div}_{n} g\right) d x: g_{i} \in C_{0}^{1}(\Omega) \forall i=1, \ldots, n+1, \sum_{i=1}^{n+1} g_{i}^{2} \leq 1\right\} .
\end{aligned}
$$


Lemma 2.1. If $v$ is smooth, $\int_{\Omega} \sqrt{v^{2}+|D v|^{2}}=\int_{\Omega} \sqrt{v^{2}+|D v|^{2}} d x$.

Proof. We consider the test function $g^{\varepsilon}=\chi_{\varepsilon}\left(v^{2}+|D v|^{2}\right)^{-1 / 2}(-D v, v)$, where $\chi_{\varepsilon}$ is a sequence of $C_{0}^{\infty}(\Omega)$ functions with $\chi_{\varepsilon} \leq 1$, converging to $\chi_{\Omega}$, the characteristic function of $\Omega$, in $L^{1}$. Then

$$
\begin{aligned}
\int_{\Omega} \sqrt{v^{2}+|D v|^{2}} & \geq \int_{\Omega} v\left(g_{n+1}^{\varepsilon}+\operatorname{div}_{n} g^{\varepsilon}\right) d x=\int_{\Omega} g_{n+1}^{\varepsilon} v-D v \cdot g^{\varepsilon} d x \\
& =\int_{\Omega} \chi_{\varepsilon} \sqrt{v^{2}+|D v|^{2}} d x,
\end{aligned}
$$

which approaches $\int_{\Omega} \sqrt{v^{2}+|D v|^{2}} d x$ as $\varepsilon \rightarrow 0$. The other direction is similar.

The next statement, while useful, is not very surprising:

Lemma 2.2. The quantity $\int_{\Omega} \sqrt{v^{2}+|D v|^{2}}$ is finite exactly when $v$ is in $\mathrm{BV}(\Omega)$.

Proof. Suppose that $v$ is in $\mathrm{BV}(\Omega)$. Then

$$
\begin{aligned}
\int_{\Omega} & \sqrt{v^{2}+|D v|^{2}} \\
& =\sup \left\{\int_{\Omega} v\left(g_{n+1}+\operatorname{div}_{n} g\right) d x: g_{i} \in C_{0}^{1}(\Omega), \sum_{i=1}^{n+1} g_{i}{ }^{2} \leq 1\right\} \\
& \leq \sup \left\{\int_{\Omega}(|v|+1) g_{n+1}+v \operatorname{div}_{n} g d x: g_{i} \in C_{0}^{1}(\Omega), \sum_{i=1}^{n+1} g_{i}{ }^{2} \leq 1\right\} \\
& \leq \sup \left\{\int_{\Omega} g_{n+1}+v \operatorname{div}_{n} g d x: g_{i} \in C_{0}^{1}(\Omega), \sum_{i=1}^{n+1} g_{i}{ }^{2} \leq 1\right\} \\
& +\sup \left\{\int_{\Omega} g_{n+1}|v| d x: g_{n+1} \in C_{0}^{1}(\Omega), \quad g_{n+1}{ }^{2} \leq 1\right\} \\
& \int_{\Omega} \sqrt{1+|D v|^{2}}+\|v\|_{L^{1}(\Omega)}<\infty .
\end{aligned}
$$

Conversely, if $\int_{\Omega} \sqrt{v^{2}+|D v|^{2}}<\infty$, then $v \in L^{1}(\Omega)$, since if not, we can take $g_{i}=0$ for $i<n+1$ and $g_{n+1}=\chi_{\varepsilon}$ (where $\chi_{\varepsilon}$ is as in Lemma 2.1) so that $\int_{\Omega} \sqrt{v^{2}+|D v|^{2}} \geq$ $\int_{\Omega}|v| \chi_{\varepsilon} d x \rightarrow \infty$ as $\varepsilon \rightarrow 0$, contradicting our assumption.

Finally we can check that

$$
\begin{aligned}
\int_{\Omega} & \sqrt{1+|D v|^{2}} \\
= & \sup \left\{\int_{\Omega} g_{n+1}+v \operatorname{div}_{n} g+v g_{n+1}-v g_{n+1} d x: g_{i} \in C_{0}^{1}(\Omega), \sum_{i=1}^{n+1} g_{i}^{2} \leq 1\right\} \\
\leq & \sup \left\{\int_{\Omega} v \operatorname{div}_{n} g+v g_{n+1} d x: g_{i} \in C_{0}^{1}(\Omega), \sum_{i=1}^{n+1} g_{i}^{2} \leq 1\right\} \\
& +\sup \left\{\int_{\Omega} g_{n+1}(1-v) d x: g_{n+1} \in C_{0}^{1}(\Omega), g_{n+1}{ }^{2} \leq 1\right\} \\
\leq & \int_{\Omega} \sqrt{v^{2}+|D v|^{2}}+\|v\|_{L^{1}(\Omega)}+|\Omega|<\infty .
\end{aligned}
$$


Corollary 2.3. If $v$ is in $\mathrm{BV}(\Omega)$ and $v_{k}$ is a mollification of $v$, then

$$
\int_{\Omega} \sqrt{v_{k}^{2}+\left|D v_{k}\right|^{2}} \rightarrow \int_{\Omega} \sqrt{v^{2}+|D v|^{2}} .
$$

This can be proved in the same manner as [Gerhardt 1974, Lemma A1]. Under the transformation $v=e^{-u}$, the wetting energy is

$$
E_{\Sigma}=-\int_{\Sigma} \beta\left(1-e^{-u}\right) d s=-\int_{\Sigma} \beta(1-v) d s,
$$

where we consider $\left.v\right|_{\Sigma}$ as a trace of $v$. As in [Giusti 1984, Theorem 2.10], if $\Sigma$ is Lipschitz, each function in $\mathrm{BV}(\Omega)$ has a trace in $L^{1}(\Sigma)$. Furthermore, if $\Sigma$ also satisfies an interior sphere condition with radius $R$, then the following estimate holds [Gerhardt 1974, Remark 2]:

$$
\int_{\Sigma}|v| d s \leq \int_{\Omega}|D v|+c_{R} \int_{\Omega}|v| d x,
$$

where $c_{R}$ depends on $n, R$, and $\Sigma$.

The integrand of $W$, the potential energy term, becomes $\int_{1}^{v} \ln h d h$ and so the complete energy, in the isothermal case, is

$$
\mathscr{F}_{1}(v)=\int_{\Omega} \sqrt{v^{2}+|D v|^{2}}+\int_{\Omega} \int_{1}^{v} \ln h d h d x-\int_{\Sigma} \beta(1-v) d s .
$$

Here we are reminded of the energy studied by Claus Gerhardt [1974], which was

$$
\int_{\Omega} \sqrt{1+|D v|^{2}}+\int_{\Omega} \int_{0}^{v} H(x, h) d h d x-\int_{\Sigma} \beta v d s
$$

for $\beta \in L^{\infty}(\Sigma)$ and $H$ satisfying the conditions (a) $\partial H / \partial h>0$, and (b) $H\left(x, h_{0}\right) \geq$ $(1+c), H\left(x,-h_{0}\right) \leq-(1+c)$ for some $h_{0} \geq 0$ and a given $c$.

\section{Height bounds}

In this section, we assume that $v$ minimizes $\mathscr{F}_{1}$ in $\mathrm{BV}(\Omega)$ and seek height bounds. A bound from above on $v$ corresponds to a bound from below on $u$, while a strictly positive bound from below on $v$ corresponds to a bound from above on $u$.

At the end of this section we show an easier way to find one-sided estimates when $\beta$ is either positive (for which we show $v$ bounded from above) or negative (for which we show $v$ bounded from below).

Height bounds from above on $v$. To estimate a minimizer $v$ from above, we follow an approach similar to those in [Gerhardt 1974; Huisken 1985] leading to a Stampacchia iteration. Note that in the original Stampacchia lemma [1966], the right-hand side of (3-1) is simply $B(k)^{\gamma}$, rather than $k B(k)^{\gamma}$. 
Lemma 3.1. Suppose B(t), nonnegative and nonincreasing in $t$, satisfies

$$
(h-k) B(h) \leq C k(B(k))^{\gamma},
$$

for all $h, k$ such that $0<k_{0} \leq k<h$, for some constants $C, k_{0}>0$ and $\gamma>1$. Then $B(K)=0$ for some sufficiently large $K$ dependent on $C, \gamma, k_{0}$ and $B\left(k_{0}\right)$.

The proof uses another lemma:

Lemma 3.2. For all $\alpha>1$ and $d>-\alpha$, the sequence

$$
s_{m}=\left(1+\frac{d}{\alpha^{m}}\right)\left(1+\frac{d}{\alpha^{m-1}}\right) \cdots\left(1+\frac{d}{\alpha}\right),
$$

converges to a nonzero limit.

Proof. We examine the sequence $\left\{\ln s_{m}\right\}$, writing each term as the partial sum $\sum_{j=1}^{m} \ln \left(1+d / \alpha^{j}\right)$ and using the ratio test for the convergence of series:

$$
\begin{aligned}
\lim _{j \rightarrow \infty} \ln \left(1+\frac{d}{\alpha^{j+1}}\right) / \ln \left(1+\frac{d}{\alpha^{j}}\right) & =\lim _{j \rightarrow \infty}\left(\frac{\partial}{\partial j} \ln \left(1+\frac{d}{\alpha^{j+1}}\right) / \frac{\partial}{\partial j} \ln \left(1+\frac{d}{\alpha^{j}}\right)\right) \\
& =\lim _{j \rightarrow \infty}\left(\frac{d \ln \alpha}{\alpha^{j+1}+d} / \frac{d \ln \alpha}{\alpha^{j}+d}\right)=\frac{1}{\alpha} .
\end{aligned}
$$

As this series converges to some limit $L,\left\{s_{m}\right\}$ converges to $e^{L}>0$.

Proof of Lemma 3.1. We begin by defining the sequence $k_{m}:=k_{0} s_{m}$, where $s_{m}$ is as in the preceding lemma, with $\alpha=2$ and $d=C\left(B\left(k_{0}\right)\right)^{\gamma-1} 2^{\gamma /(\gamma-1)}>0$ (we assume here that $B\left(k_{0}\right) \neq 0$, otherwise the lemma is trivially true). Note that as $k_{m+1}-k_{m}=k_{0} d 2^{-(m+1)}>0,\left\{k_{m}\right\}$ is positive and increasing, and, by the above result, converges to some limit $K$.

We now prove that $B\left(k_{m}\right) \leq B\left(k_{0}\right) 2^{\mu m}$ for $\mu=(1-\gamma)^{-1}<0$, by induction.

The base step, for $m=1$, is as follows: by assumption (3-1),

$$
\left(k_{1}-k_{0}\right) B\left(k_{1}\right) \leq C k_{0} B\left(k_{0}\right)^{\gamma},
$$

and so using $k_{1}-k_{0}=k_{0} d / 2$ we find that

$$
B\left(k_{1}\right) \leq C \frac{2^{1-\mu}}{d} B\left(k_{0}\right)^{\gamma-1} B\left(k_{0}\right) 2^{\mu}=2^{1-\mu-\gamma /(\gamma-1)} B\left(k_{0}\right) 2^{\mu}=B\left(k_{0}\right) 2^{\mu} .
$$

Now we make the inductive assumption that $B\left(k_{m}\right) \leq B\left(k_{0}\right) 2^{\mu m}$. We use this and condition (3-1) to estimate

$$
\begin{aligned}
B\left(k_{m+1}\right) & \leq C \frac{2^{m+1}}{d} B\left(k_{m}\right)^{\gamma} \leq C \frac{2^{m+1}}{d}\left(B\left(k_{0}\right) 2^{\mu m}\right)^{\gamma} \\
& =2^{m+1-\gamma /(\gamma-1)} B\left(k_{0}\right)^{1-\gamma}\left(B\left(k_{0}\right) 2^{\mu m}\right)^{\gamma} \leq B\left(k_{0}\right) 2^{\mu(m+1)} .
\end{aligned}
$$

By the monotonicity of $B, B(K) \leq \lim _{m \rightarrow \infty} B\left(k_{m}\right) \leq \lim _{m \rightarrow \infty} B\left(k_{0}\right) 2^{\mu m}=0$. 
Theorem 3.3. Suppose that $v$ minimizes $\mathscr{F}_{1}$ in $\mathrm{BV}^{+}(\Omega)$. Then there exists a bound from above on $v, v \leq c, L^{1}$-almost everywhere.

Proof. We set $A(k)=\{x \in \Omega: v(x)>k\}$. The goal is to show that the nonincreasing $|A(k)|$ vanishes for some large $k$. Let $w:=\min (v, k)$. As $v$ minimizes $\mathscr{F}_{1}$, we have $\mathscr{F}_{1}(v) \leq \mathscr{F}_{1}(w)$, which after rearranging and taking into account that $w \in \mathrm{BV}(\Omega)$, that $D w=D v$ in $\Omega \backslash A(k)$, and that $D w=0$ in $A(k)$, gives

$$
0 \geq\left\{\int_{A(k)} \sqrt{v^{2}+|D v|^{2}}-\int_{A(k)} k d x\right\}+\int_{A(k)} \int_{k}^{v} \ln h d h d x+\int_{\Sigma} \beta(v-w) d s .
$$

We estimate the boundary term using (2-1):

$$
\left|\int_{\Sigma} \beta(v-w) d s\right| \leq(1-a)\left(\int_{\Omega}|D(v-w)|+c_{R} \int_{\Omega}|v-w| d x\right) .
$$

One can easily show that $\int_{\Omega}|D v| \leq \int_{\Omega} \sqrt{v^{2}+|D v|^{2}}$, so that (3-2) gives

$$
k|A(k)| \geq a \int_{A(k)}|D(v-k)|+\left(\ln k-(1-a) c_{R}\right) \int_{A(k)}|v-k| d x .
$$

For BV functions on Lipschitz domains $\Omega$, we have the Sobolev inequality

$$
\left(\int_{\Omega}|f|^{n /(n-1)} d x\right)^{(n-1) / n} \leq c_{\Omega}\left(\int_{\Omega}|D f|+\int_{\Omega}|f| d x\right) ;
$$

see, for example, [Giusti 1984, Theorem 1.28]. We rearrange this inequality as

$$
a \int_{\Omega}|D f| \geq \frac{a}{c_{\Omega}}\left(\int_{\Omega}|f|^{n /(n-1)} d x\right)^{(n-1) / n}-a \int_{\Omega}|f| d x .
$$

Using the estimate above with $f=v-w,(3-3)$ becomes

$k|A(k)| \geq \frac{a}{c_{\Omega}}\left(\int_{\Omega}|v-w|^{n /(n-1)} d x\right)^{(n-1) / n}+\left(\ln k-(1-a) c_{R}-a\right) \int_{A(k)}|v-w| d x$.

By choosing $k$ large enough, we can ensure that the quantity $\ln k-(1-a) c_{R}-a$ is positive, and drop this term altogether. The Hölder inequality gives

$$
\|f\|_{L^{n /(n-1)}(A(k))} \geq\|f\|_{L^{1}(A(k))}|A(k)|^{-1 / n},
$$

and so for all $h, k$ with $h>k \geq k_{0}$ for a certain choice of $k_{0}$, we have

$$
\frac{a}{c_{\Omega}}(h-k)|A(h)| \leq \frac{a}{c_{\Omega}} \int_{A(k)}(v-k) d x \leq k|A(k)|^{1+1 / n} .
$$

Now we apply Lemma 3.1 and find that $|A(K)|=0$ for sufficiently large $K$. 
Height bounds on $v$ from below. The proof is similar to that of Theorem 3.3. However, a different Stampacchia-type result is needed, with a yet weaker condition on the right-hand side.

Theorem 3.4. Suppose that $v$ minimizes $\mathscr{F}_{1}$ in $\mathrm{BV}^{+}(\Omega)$. Then there exists a bound from below on $v, 0 \leq c \leq v, L^{1}$-almost everywhere.

Lemma 3.5. Suppose $B(t)$, nonnegative and nonincreasing in $t$, satisfies

$$
(h-k) B(h) \leq C h(B(k))^{\gamma},
$$

for all $h, k$ such that $0<k_{0} \leq k<h$, for some constants $C, k_{0}>0$ and $\gamma>1$. If

$$
C B\left(k_{0}\right)^{\gamma-1}<1,
$$

then there exists a $K<\infty$ such that $B(K)=0$.

Proof. From (3-5), we may choose $d>0$ and $\alpha>1$ such that $C B\left(k_{0}\right)^{\gamma-1} \alpha^{\gamma /(\gamma-1)} \leq$ $d<\alpha$.

Next, define the sequence $k_{m}:=k_{0} / s_{m}=k_{m-1}\left(1-d / \alpha^{m}\right)^{-1}$, where $s_{m}$ is as in Lemma 3.2 (but note the change of sign on $d$ ); here $\left\{k_{m}\right\}$ is positive, increasing, and by Lemma 3.2, convergent to some $K$.

We now prove that $B\left(k_{m}\right) \leq B\left(k_{0}\right) \alpha^{-m /(\gamma-1)}$ by induction.

The base step, for $m=1$, is as follows: by the definition of the sequence, we have $k_{1}=k_{0}(1-d / \alpha)^{-1}$, and so our assumption (3-4) gives

$$
\left(k_{1}-k_{0}\right) B\left(k_{1}\right) \leq C k_{1} B\left(k_{0}\right)^{\gamma},
$$

which leads to

$$
B\left(k_{1}\right) \leq \frac{C \alpha}{d} B\left(k_{0}\right)^{\gamma} \leq B\left(k_{0}\right) \alpha^{-1 /(\gamma-1)} .
$$

Now we make the inductive assumption that $B\left(k_{m}\right) \leq B\left(k_{0}\right) \alpha^{-m /(\gamma-1)}$, and show that this then holds for $k_{m+1}$ : we use (3-4) to estimate

$$
\begin{aligned}
B\left(k_{m+1}\right) & \leq C \frac{k_{m+1}}{k_{m+1}-k_{m}} B\left(k_{m}\right)^{\gamma} \\
& \leq C \frac{\alpha^{m+1}}{d} B\left(k_{0}\right)^{\gamma-1} \alpha^{\frac{-m \gamma}{\gamma-1}+\frac{m+1}{\gamma-1}}\left[B\left(k_{0}\right) \alpha^{\frac{-(m+1)}{\gamma-1}}\right] \leq B\left(k_{0}\right) \alpha^{\frac{-(m+1)}{\gamma-1}} .
\end{aligned}
$$

Finally, the monotonicity of $B$ implies that

$$
B(K) \leq \lim _{m \rightarrow \infty} B\left(k_{m}\right) \leq \lim _{m \rightarrow \infty} B\left(k_{0}\right) \alpha^{-m /(\gamma-1)}=0 .
$$

We will need to show that the measure of the set where $v$ is small is small enough to satisfy (3-5). This is the only place in this paper where it is not immediately clear that rescaling the constants $\gamma_{i}$ to 1 does not result in a loss of generality. Consequently, we include the arbitrary weightings in $\mathscr{F}_{1}$ in the following step. 
Lemma 3.6. Let $v$ minimize $\mathscr{g}_{1}=\gamma_{1} E_{\varphi}+\gamma_{2} W+\gamma_{3} E_{\Sigma}$ in $\mathrm{BV}^{+}(\Omega)$, and set

$$
\tilde{A}(k):=\{x \in \Omega: v(x)<1 / k\} .
$$

Then for all $\eta>0$ we can find a $k$ such that $|\tilde{A}(k)| \leq \eta$.

Proof. Define the comparison function $w:=\max \{v, 1 / k\} \in \mathrm{BV}^{+}(\Omega)$ for any $k \geq k_{0}$, $k_{0}$ to be chosen later. Note that $0 \leq w-v \leq 1 / k$. Since $v$ minimizes $\mathscr{F}_{1}$, we have $\mathscr{F}_{1}(v) \leq \mathscr{F}_{1}(w)$. We use $\int \sqrt{u^{2}+|D u|^{2}} \geq \int|u| d x$ for $u \in \mathrm{BV}^{+}(\Omega)$ to estimate

$$
\begin{aligned}
& 0 \geq \mathscr{g}_{1}(v)-\mathscr{g}_{1}(w) \\
&=\gamma_{1} \int_{\tilde{A}(k)} \sqrt{v^{2}+|D v|^{2}}-\gamma_{1} \int_{\tilde{A}(k)} \frac{1}{k} d x-\gamma_{3} \int_{\Sigma} \beta(w-v) d s+\gamma_{2} \int_{\tilde{A}(k)} v \ln v-v-\frac{1}{k} \ln \frac{1}{k}+\frac{1}{k} d x \\
& \geq \int_{\tilde{A}(2 k)}-\left|\gamma_{1}-\gamma_{2}\right|\left|v-\frac{1}{k}\right|+\gamma_{2}\left(v \ln v-\frac{1}{k} \ln \frac{1}{k}\right) d x \\
&+\int_{\tilde{A}(k) \backslash \tilde{A}(2 k)}-\left|\gamma_{1}-\gamma_{2}\right|\left|v-\frac{1}{k}\right|+\gamma_{2}\left(v \ln v-\frac{1}{k} \ln \frac{1}{k}\right) d x \\
&-\gamma_{3}(1-a)|\Sigma| \frac{1}{k}
\end{aligned}
$$

(now choose $k_{0}$ large enough so that $x \ln x$ is decreasing for $0<x \leq 1 / k_{0}$ )

$$
\begin{aligned}
& \geq \int_{\tilde{A}(2 k)}-\left|\gamma_{1}-\gamma_{2}\right| \frac{1}{k}+\gamma_{2}\left(\frac{1}{2 k} \ln \frac{1}{2 k}-\frac{1}{k} \ln \frac{1}{k}\right) d x+\int_{\tilde{A}(k) \backslash \tilde{A}(2 k)}-\left|\gamma_{1}-\gamma_{2}\right| \frac{1}{2 k} d x \\
& -\gamma_{3}(1-a)|\Sigma| \frac{1}{k} \\
& \geq-\left|\gamma_{1}-\gamma_{2}\right| \frac{2}{k}|\Omega|-\gamma_{3}(1-a)|\Sigma| \frac{1}{k}+\gamma_{2} \int_{\tilde{A}(2 k)}\left(-\frac{1}{2 k}\right)(\ln \xi+1) d x \\
& \text { for some } \xi \in\left(\frac{1}{2 k}, \frac{1}{k}\right) \\
& \geq-\left|\gamma_{1}-\gamma_{2}\right| \frac{2}{k}|\Omega|-\gamma_{3}(1-a)|\Sigma| \frac{1}{k}+\gamma_{2}|\tilde{A}(2 k)|\left(-\frac{1}{2 k}\right)\left(\ln \frac{1}{2 k}+1\right) .
\end{aligned}
$$

Rearranging, and choosing $k_{0}$ large enough that $\ln \left(2 k_{0}\right)>1$, we find that

$$
|\tilde{A}(2 k)| \leq \frac{4\left|\gamma_{1}-\gamma_{2}\right||\Omega|+\gamma_{3}(1-a)|\Sigma|}{\gamma_{2}(\ln 2 k-1)}<\eta
$$

for sufficiently large $k>k_{0}$.

Proof of Theorem 3.4. Let $\tilde{A}(k)$ be defined as above. Set $w:=\max (v, 1 / k)$, for some $k \geq k_{0}$. Again, as $v$ minimizes $\mathscr{F}_{1}$, then $\mathscr{F}_{1}(v) \leq \mathscr{F}_{1}(w)$. Proceeding exactly as in the proof of Theorem 3.3, we obtain 


$$
\begin{aligned}
0 & \geq a \int_{\Omega}|D(v-w)|-\frac{1}{k}|\tilde{A}(k)|-\left((1-a) c_{R}+\ln (1 / k)\right) \int_{\Omega}|v-w| d x \\
& \geq \frac{a}{c_{\Omega}}\|v-w\|_{L^{n /(n-1)}(\Omega)}-\frac{1}{k}|\tilde{A}(k)|-\left((1-a) c_{R}+\ln (1 / k)+a\right) \int_{\Omega}|v-w| d x,
\end{aligned}
$$

and if we choose $k_{0}$ large so that $\ln k \geq c_{R}(1-a)+a$, then the final term above is positive. We drop it and apply the Hölder inequality to the $L^{n /(n-1)}$ term, leaving us with $(1 / k)|\tilde{A}(k)| \geq\left(a / c_{\Omega}\right)\|v-w\|_{L^{1}(\tilde{A}(k))}|\tilde{A}(k)|^{-1 / n}$, and so for each $h>k \geq k_{0}$ we have

$$
(h-k)|\tilde{A}(h)| \leq C h|\tilde{A}(k)|^{1+1 / n} .
$$

Lemma 3.6 implies that we can find $k_{0}$ large enough that $C \tilde{A}\left(k_{0}\right)^{1 / n}<1$. We can then apply the Stampacchia-type Lemma 3.5 to conclude that $|\tilde{A}(K)|=0$ for large $K$, and so $v \geq 1 / K$ almost everywhere.

Height estimates in the cases $\beta \leq 0$ and $\beta \geq 0$. Height estimates are easier to obtain in case $\beta$ is either nonpositive or nonnegative.

We begin by observing a height bound for $v$ in the surface energy term. This closely follows [Bemelmans and Dierkes 1987, Lemma 5], and may be proved in the same way.

Lemma 3.7. Let $v \in \mathrm{BV}^{+}(\Omega)$ and suppose that $A(k)=\{x \in \Omega: v(x)>k\}$ has positive measure. Then $w=\min (v, k) \in \mathrm{BV}^{+}(\Omega)$ and for almost all $k$,

$$
\int_{\Omega} \sqrt{w^{2}+|D w|^{2}}<\int_{\Omega} \sqrt{v^{2}+|D v|^{2}} .
$$

Theorem 3.8. Suppose that $v \in \mathrm{BV}^{+}(\Omega)$ minimizes $\mathscr{F}_{1}$, and that $\beta \geq 0$. Then $v$ is bounded from above.

Proof. Set $w=\min (v, k)$. Suppose that $A(k)$ is of positive measure for some $k \geq 1$. We may choose $k$ so that Lemma 3.7 gives us

$$
\int_{\Omega} \sqrt{w^{2}+|D w|^{2}}-\int_{\Omega} \sqrt{v^{2}+|D v|^{2}}<0 .
$$

We note that

$$
\begin{aligned}
\int_{\Omega} \int_{1}^{w} \ln h d h d x-\int_{\Omega} \int_{1}^{v} \ln h d h d x & =\int_{\Omega} \int_{v}^{w} \ln h d h d x \\
& =\int_{\Omega \cap\{x: v(x) \geq k\}} \int_{v}^{k} \ln h d h d x \leq 0 .
\end{aligned}
$$

Finally,

$$
-\int_{\Sigma} \beta(1-w) d s+\int_{\Sigma} \beta(1-v) d s=\int_{\Sigma} \beta(w-v) d s \leq 0
$$


if $\beta \geq 0$. Together, these inequalities give $\mathscr{F}_{1}(w)-\mathscr{F}_{1}(v)<0$, contradicting that $v$ was a minimum. It follows that $|A(k)|$ cannot be positive, and so $v \leq k$.

Lemma 3.9. Suppose that $v$ minimizes $\mathscr{F}_{1}$, and $\beta \leq 0$. Then $v \geq e^{-1}$.

Proof. Set $w=\max (v, \varepsilon)$, and write $\tilde{A}(\varepsilon)=\{x \in \Omega: v(x)<\varepsilon\}$. Then

$$
\begin{aligned}
& \mathscr{F}_{1}(v)-\mathscr{F}_{1}(w) \\
& =\int_{\tilde{A}(\varepsilon)} \sqrt{v^{2}+|D v|^{2}}-\int_{\tilde{A}(\varepsilon)} \sqrt{w^{2}+|D w|^{2}}-\int_{\Omega} \int_{v}^{\max (v, \varepsilon)} \ln h d h d x-\int_{\Sigma} \beta(w-v) d s \\
& =\int_{\tilde{A}(\varepsilon)} \sqrt{v^{2}+|D v|^{2}}-\int_{\tilde{A}(\varepsilon)} \varepsilon d x-\int_{\tilde{A}(\varepsilon)} \int_{v}^{\varepsilon} \ln h d h d x-\int_{\Sigma} \beta(\max (v, \varepsilon)-v) d s \\
& \geq \int_{\tilde{A}(\varepsilon)} \sqrt{v^{2}+|D v|^{2}}-\int_{\tilde{A}(\varepsilon)}(\varepsilon-v+v) d x+(-\ln \varepsilon) \int_{\tilde{A}(\varepsilon)}(\varepsilon-v) d x \\
& =\int_{\tilde{A}(\varepsilon)} \sqrt{v^{2}+|D v|^{2}}-\int_{\tilde{A}(\varepsilon)} v d x+(-\ln \varepsilon-1) \int_{\tilde{A}(\varepsilon)}(\varepsilon-v) d x>0
\end{aligned}
$$

for all $\varepsilon<e^{-1}$, if $\int_{\tilde{A}(\varepsilon)}|\varepsilon-v| \neq 0$. However, this would contradict our assumption that $v$ is minimal for $\mathscr{F}_{1}$, so we conclude that $|\tilde{A}(\varepsilon)|=0$ for small enough $\varepsilon$.

\section{Existence of a minimizer}

Lemma 4.1 (Lower bounds for the energy). If $v \in \mathrm{BV}^{+}(\Omega)$, then

$$
\mathscr{g}_{1}(v) \geq C(n, R, a,|\Omega|),
$$

where $C$ is not necessarily positive.

Proof. As before, we can incorporate the wetting energy into the surface tension term using (2-1), so that

$$
\begin{aligned}
\mathscr{E}_{1}(v) & \geq \int_{\Omega} \sqrt{v^{2}+|D v|^{2}}+\int_{\Omega} \int_{1}^{v} \ln h d h d x \\
& \geq a \int_{\Omega}|D v|+\int_{\Omega} f(v) d x,
\end{aligned}
$$

where $f(v):=v(\ln v-1)+1-c_{R}(1-a)|1-v|$ is bounded below by a constant dependent on $c_{R}$ and $a$. The result follows.

We define a minimizing sequence for $\mathscr{F}_{1}$ as a sequence $v_{j} \in \mathrm{BV}^{+}(\Omega)$ with

$$
\lim _{j \rightarrow \infty} \mathscr{F}_{1}\left(v_{j}\right)=\inf _{w \in \mathrm{BV}^{+}(\Omega)} \mathscr{F}_{1}(w)=: m .
$$

Lemma 4.2. A minimizing sequence for $\mathscr{F}_{1}$ is uniformly bounded in the BV-norm. 
Proof. We can assume that $\mathscr{F}_{1}\left(v_{j}\right) \leq m+1$ for $j$ large enough. As in the previous lemma, where we defined $f$, we then have

$$
m+1 \geq \mathscr{F}_{1}\left(v_{j}\right) \geq a \int_{\Omega}\left|D v_{j}\right|+\int_{\Omega} f\left(v_{j}\right) d x,
$$

so the uniform bound follows from the lower bound on $f$ :

$$
\int_{\Omega}\left|D v_{j}\right| \leq \frac{1}{a}\left(m+1-|\Omega| \inf _{h \in \mathbb{R}^{+}} f(h)\right) .
$$

Also, since there exist positive constants $\alpha_{1}, \alpha_{2}$ such that $f(t) \geq \alpha_{1} t-\alpha_{2}$, we have the uniform $L^{1}$ bound

$$
\left\|v_{j}\right\|_{L^{1}(\Omega)} \leq \frac{1}{\alpha_{1}}\left(\int_{\Omega}\left(f\left(v_{j}\right)+\alpha_{2}\right) d x\right) \leq \frac{1}{\alpha_{1}}\left(m+1+\alpha_{2}|\Omega|\right) .
$$

Lemma 4.3 (Lower semicontinuity of $\mathscr{F}_{1}$ ). A sequence $v_{k} \in \mathrm{BV}^{+}(\Omega)$ with $v_{k} \rightarrow v$ in $L^{1}(\Omega)$ satisfies

$$
\mathscr{F}_{1}(v) \leq \liminf _{k \rightarrow \infty} \mathscr{E}_{1}\left(v_{k}\right) .
$$

Proof. We show that the surface energy term is lower semicontinuous. For any admissible $g$, we have

$$
\begin{aligned}
\int_{\Omega} v\left(g_{n+1}+\operatorname{div}_{n} g\right) d x & =\lim _{k \rightarrow \infty} \int_{\Omega} v_{k}\left(g_{n+1}+\operatorname{div}_{n} g\right) d x \\
& =\liminf _{k \rightarrow \infty} \int_{\Omega} v_{k}\left(g_{n+1}+\operatorname{div}_{n} g\right) d x \\
& \leq \liminf _{k \rightarrow \infty} \int_{\Omega} \sqrt{v_{k}^{2}+\left|D v_{k}\right|^{2}} .
\end{aligned}
$$

Lower semicontinuity follows by taking the supremum over all admissible $g$.

Continuity of the remaining terms of $\mathscr{F}_{1}$ follows as in [Gerhardt 1974, Appendix II].

Combining all of the above results we have:

Theorem 4.4 (Existence of a minimizer). There exists a function $v \in \mathrm{BV}^{+}(\Omega)$, such that

$$
\mathscr{F}_{1}(v)=\inf _{w \in \mathrm{BV}^{+}(\Omega)} \mathscr{F}_{1}(w) .
$$

Proof. Let $\left\{v_{j}\right\}$ be the minimizing sequence of Lemma 4.2, with $\left\|v_{j}\right\|_{\mathrm{BV}(\Omega)} \leq C$. By the standard compactness theorem (for example [Giusti 1984, Theorem 1.19]) there exists a subsequence $v_{j^{\prime}} \rightarrow v$ in $L^{1}(\Omega)$.

Since the BV-norm is lower semicontinuous, $v$ is also in $\mathrm{BV}^{+}(\Omega)$, and $\mathscr{F}_{1}(v) \geq$ $\inf _{v \in \mathrm{BV}^{+}(\Omega)} \mathscr{F}_{1}(w)=m$. Lower semicontinuity of $\mathscr{F}_{1}$, as in Lemma 4.3, gives $\mathscr{F}_{1}(v) \leq \liminf \mathscr{F}_{1}\left(v_{j^{\prime}}\right)=m$, completing the proof. 


\section{Regularity}

We now show, using a procedure similar to [Gerhardt 1974], that a minimizer $v \in \mathrm{BV}^{+}(\Omega)$ of $\mathscr{F}_{1}$ is locally Lipschitz in $\Omega$. Then we show that the corresponding minimizer $u=-\ln v$ of the energy $\mathscr{F}$ is a variational solution in the sense of [Athanassenas and Finn 2006], so the results of that paper yield regular solutions $u$.

Theorem 5.1. The energy $\Phi_{1}$ has a locally Lipschitz minimizer in $\mathrm{BV}^{+}(\Omega)$.

Proof. Let $v$ be a minimizer of $\mathscr{g}_{1}$ in $\mathrm{BV}^{+}(\Omega)$. We mollify $v$ over the whole of $\Omega$. The mollification $v_{\varepsilon}$ is in $C^{\infty}(\Omega)$, and shares the height bounds derived for $v$ in Section 3 (that is, bounded above and bounded from below away from zero). Furthermore, since $v \in \mathrm{BV}(\Omega)$,

$$
v_{\varepsilon} \rightarrow v \text { in } L^{1}(\Omega) \quad \text { and } \quad \int_{\Omega}\left|D v_{\varepsilon}\right| \rightarrow \int_{\Omega}|D v| .
$$

Corollary 2.3 for the surface energy and standard convergence results for the remaining energy terms then imply that

$$
\mathscr{F}_{1}\left(v_{\varepsilon}\right) \rightarrow \mathscr{F}_{1}(v) .
$$

Let $B \subset \Omega$ be any ball of sufficiently small radius $\rho$, and consider the following two related Dirichlet problems:

$$
\begin{cases}\operatorname{div} \frac{D w_{\varepsilon}}{\sqrt{w_{\varepsilon}^{2}+\left|D w_{\varepsilon}\right|^{2}}}=\frac{w_{\varepsilon}}{\sqrt{w_{\varepsilon}^{2}+\left|D w_{\varepsilon}\right|^{2}}}+\ln w_{\varepsilon} & \text { in } B, \\ w_{\varepsilon}=v_{\varepsilon} & \text { on } \partial B\end{cases}
$$

and

$$
\begin{cases}\operatorname{div} \frac{D u_{\varepsilon}}{\sqrt{1+\left|D u_{\varepsilon}\right|^{2}}}=\sigma\left(-\frac{1}{\sqrt{1+\left|D u_{\varepsilon}\right|^{2}}}+u_{\varepsilon}\right) & \text { in } B, \\ u_{\varepsilon}=-\sigma \ln \left(v_{\varepsilon}\right) & \text { on } \partial B .\end{cases}
$$

The second expression is in fact a family of problems, indexed by $\sigma \in[0,1]$. This family is of mean curvature type. Note that for smooth $w_{\varepsilon}$ and $u_{\varepsilon},(5-2)$ is equivalent to (5-3) for $\sigma=1$, with the correspondence $w_{\varepsilon}=e^{-u_{\varepsilon}}$.

Our next step is to solve (5-3) for $\sigma=1$ using the continuity method. We apply [Gilbarg and Trudinger 2001, Theorem 13.8]. A prerequisite for this is to show that a smooth solution $u^{\sigma}$ of (5-3), for any $\sigma \in[0,1]$, has height and gradient bounds independent of $\sigma$.

The height bound may be found in [Serrin 1969]; however, the geometric nature of our problem admits a shorter proof, which we present as the following lemma. Lemma 5.2. Let $u^{\sigma}$ be a smooth solution to (5-3) corresponding to $\sigma \in[0,1]$. Then, for some $M_{1}$ depending only on $\sup _{\Omega}\left|\ln v_{\varepsilon}\right|$, we have

$$
\sup _{B}\left|u^{\sigma}\right|<M_{1} \text {. }
$$


Proof. Suppose that $u^{\sigma}$ achieves a positive interior maximum, $u^{\sigma}(\tilde{x})=\widetilde{M}$ at some point $\tilde{x} \in B$. If $\tilde{M}>1$, then the mean curvature $H\left(u^{\sigma}\right)=\operatorname{div}\left(D u^{\sigma} / \sqrt{1+\left|D u^{\sigma}\right|^{2}}\right)$ at $\tilde{x}$ must be strictly positive. But a point of positive mean curvature cannot correspond to an interior maximum, contradicting the assumption $\widetilde{M}>1$. We conclude that

$$
u^{\sigma} \leq \min \left\{1, \sup _{\partial B}\left|\sigma \ln v_{\varepsilon}\right|\right\} \leq \min \left\{1, \sup _{\partial B}\left|\ln v_{\varepsilon}\right|\right\} .
$$

Similarly, $u^{\sigma}$ has no negative internal minimum, so $u^{\sigma} \geq-\sup _{\partial B}\left|\ln v_{\varepsilon}\right|$.

We continue with the proof of Theorem 5.1. We find that the gradient bound

$$
\sup _{B}\left|D u^{\sigma}\right| \leq M_{2}
$$

is an application of standard results. Firstly, an interior gradient bound can either be derived by applying a maximum principle to the elliptic equation satisfied by the gradient; or by using [Ladyzhenskaya and Ural'tseva 1970, Theorem 4], which gives

$$
\sup _{B^{\prime}}\left|D u^{\sigma}\right| \leq M_{3}
$$

where $B^{\prime} \Subset B$ and $M_{3}$ is dependent on $\operatorname{dist}\left(B^{\prime}, \partial B\right), n$ and $\sup \left|u^{\sigma}\right|$.

Secondly, a boundary gradient estimate

$$
\sup _{\partial B}\left|D u^{\sigma}\right| \leq M_{4}
$$

results from Corollary 14.5 with the structure condition (14.33) of [Gilbarg and Trudinger 2001]. Here $M_{4}$ is dependent on $\left|\ln v_{\varepsilon}\right|_{C^{2}(\partial B)}, n$, sup $\left|u^{\sigma}\right|$, and $\rho$. Together these two gradient estimates give us (5-5).

The conditions for the continuity method being satisfied, the problem (5-3), with $\sigma=1$, has a $C^{2, \alpha}(B)$ solution which we call $u_{\varepsilon}$. It has height and gradient bounds (5-4) and (5-5). It is also unique: the proof is similar to that of Theorem 2.2 in [Athanassenas and Finn 2006], adjusted to Dirichlet boundary data.

We set $w_{\varepsilon}=e^{-u_{\varepsilon}}$. This is a $C^{2, \alpha}(B)$ solution of (5-2) with height bound $e^{-M_{1}} \leq$ $w_{\varepsilon} \leq e^{M_{1}}$ and gradient bound $\left|D w_{\varepsilon}\right| \leq M_{2} e^{M_{1}}$.

Note that (5-2) is the Euler-Lagrange equation for the energy

$$
\mathscr{f}_{2}(w):=\int_{B} \sqrt{w^{2}+|D w|^{2}}+\int_{B} \int_{1}^{w} \ln h d h d x,
$$

and so $w_{\varepsilon}$ is a critical point of $\mathscr{F}_{2}$ in the class of $H^{1,2}(B)$ functions with boundary data $v_{\varepsilon}$. Furthermore, as the integrand of $\mathscr{F}_{2}$ is convex in $(w, D w), w_{\varepsilon}$ is also a minimizer in this class (see, for example, the remark in Section 8.2.3 of [Evans $1998])$ and hence in the smaller set $C^{2, \alpha}(B)$. 
In particular, if we compare $w_{\varepsilon}$ to $v_{\varepsilon}$, we have

$$
\begin{aligned}
\int_{B} \sqrt{w_{\varepsilon}^{2}+\left|D w_{\varepsilon}\right|^{2}}+\int_{B} \int_{1}^{w_{\varepsilon}} & \ln h d h d x \\
& \leq \int_{B} \sqrt{v_{\varepsilon}^{2}+\left|D v_{\varepsilon}\right|^{2}}+\int_{B} \int_{1}^{v_{\varepsilon}} \ln h d h d x .
\end{aligned}
$$

Now let $\tilde{v}_{\varepsilon}$ be defined by

$$
\tilde{v}_{\varepsilon}= \begin{cases}w_{\varepsilon} & \text { in } B, \\ v_{\varepsilon} & \text { in } \Omega \backslash B .\end{cases}
$$

Using (5-7) for the region $B$ where $\tilde{v}_{\varepsilon}$ may be different to $v_{\varepsilon}$, we see that $\mathscr{g}_{1}\left(\tilde{v}_{\varepsilon}\right) \leq$ $\mathscr{F}_{1}\left(v_{\varepsilon}\right)$.

Now we will show that $\tilde{v}_{\varepsilon}$ converges to a $\mathrm{BV}(\Omega)$ function which is locally Lipschitz.

Uniform $L^{1}(\Omega \backslash B)$ bounds are given by the height bounds for $v$ in Section 3 . Uniform $L^{1}(B)$ bounds are given by $\sup _{B}\left|w_{\varepsilon}\right| \leq e^{M_{1}}$ where $M_{1}$ is the constant in (5-4); $M_{1}$ also depends on the height bounds for $v$.

As a consequence of (5-1), we may assume that $\mathscr{F}_{1}\left(v_{\varepsilon}\right) \leq \mathscr{F}_{1}(v)+1$. Then $\mathscr{F}_{1}\left(\tilde{v}_{\varepsilon}\right) \leq \mathscr{F}_{1}(v)+1$, and so

$$
\begin{aligned}
\int_{\Omega} \sqrt{\tilde{v}_{\varepsilon}^{2}+\left|D \tilde{v}_{\varepsilon}\right|^{2}} \leq \mathscr{\Phi}_{1}(v)+1-\int_{\Omega} \int_{1}^{\tilde{v}_{\varepsilon}} \ln h d h d x & +\int_{\Sigma} \beta\left(1-\tilde{v}_{\varepsilon}\right) d s \\
\leq & \mathscr{\Phi}_{1}(v)+1+|\Omega| \sup _{\inf v \leq h \leq \sup v}(h \ln h-h+1) \\
& +|\Sigma| \sup _{\Sigma}|\beta|\left(1+\sup _{\Omega}|v|\right)
\end{aligned}
$$

which is bounded above, independently of $\varepsilon$. Uniform BV bounds follow as in Lemma 2.2. Therefore a subsequence of $\tilde{v}_{\varepsilon}$ converges to $v_{0} \in \mathrm{BV}(\Omega)$, and $v_{0}$ is Lipschitz in $B^{\prime}$ with bounds given by (5-6).

Lower semicontinuity of the functional now gives

$$
\mathscr{F}_{1}\left(v_{0}\right) \leq \liminf \mathscr{F}_{1}\left(\tilde{v}_{\varepsilon}\right) \leq \liminf \mathscr{F}_{1}\left(v_{\varepsilon}\right)=\mathscr{F}_{1}(v)
$$

but as $v$ was assumed to minimize $\mathscr{F}_{1}$ these must all be equal.

We can cover the interior of $\Omega$ with such sets $B^{\prime}$, and by repeating the argument above for each of them, we conclude that there exists a minimizer of $\mathscr{F}_{1}$ that is locally Lipschitz on interior sets.

Reconsidering the problem of a capillary surface $\mathscr{S}=\operatorname{graph} u$ that minimizes the original energy functional $\mathscr{g}$ given in the introduction, we conclude that the found $v$ corresponds to a minimizer in the class $\left\{w: e^{-w} \in \mathrm{BV}^{+}(\Omega)\right\}$. This solution is given by $u=-\ln v$, and is locally Lipschitz on interior sets. 
We move on to show that under the condition $v \in H^{1,1}(\Omega)$, the corresponding $u$ is a variational solution, satisfying (5-9), which will be used in the regularity proof. Theorem 5.3. A minimizer $v$ of $\mathscr{F}_{1}$ in $H^{1,1}(\Omega)$ is a variational solution in the sense of [Athanassenas and Finn 2006], that is, satisfies the equation

$$
\begin{aligned}
\int_{\Omega} \frac{D v}{\sqrt{v^{2}+|D v|^{2}}} \eta d x+\int_{\Omega} \frac{v}{\sqrt{v^{2}+|D v|^{2}}} & \eta d x \\
& +\int_{\Omega} \ln v \eta d x+\int_{\partial \Omega} \beta \eta d s=0,
\end{aligned}
$$

for all $\eta \in L^{\infty} \cap H^{1,1}(\Omega)$.

Proof. (See also [Finn and Gerhardt 1977, Lemma 2, Section 3].) Since $v$ minimizes $\mathscr{I}_{1}$,

$$
\mathscr{F}_{1}(v+\epsilon \eta)-\mathscr{F}_{1}(v) \geq 0
$$

holds for any $\eta \in H^{1,1}(\Omega)$ and $\epsilon \in \mathbb{R}$. We have

$$
\begin{aligned}
\mathscr{F}_{1}(v+\epsilon \eta)-\mathscr{F}_{1}(v)= & \int_{\Omega}\left(\sqrt{(v+\epsilon \eta)^{2}+|D(v+\epsilon \eta)|^{2}}-\sqrt{v^{2}+|D v|^{2}}\right) d x \\
& +\int_{\Omega} \int_{v}^{v+\epsilon \eta} \ln h d h+\int_{\partial \Omega} \beta \epsilon \eta d s d x \\
= & \int_{\Omega} \int_{0}^{\epsilon} \frac{D(v+\lambda \eta)}{\sqrt{(v+\lambda \eta)^{2}+\mid D\left(v+\left.\lambda \eta\right|^{2}\right.}} \cdot D \eta d \lambda d x \\
& +\int_{\Omega} \int_{0}^{\epsilon} \frac{(v+\lambda \eta)}{\sqrt{(v+\lambda \eta)^{2}+|D(v+\lambda \eta)|^{2}}} \eta d \lambda d x \\
& +\int_{\Omega} \int_{0}^{\epsilon} \ln (v+\lambda \eta) \eta d \lambda d x+\int_{\partial \Omega} \beta \epsilon \eta d s,
\end{aligned}
$$

after adding and subtracting $\int_{\Omega} \sqrt{v^{2}+|D(v+\epsilon \eta)|^{2}}$, and with $\theta \in[v, v+\epsilon \eta]$.

We note that

$$
\begin{aligned}
& \lim _{\lambda \rightarrow 0} \frac{D(v+\lambda \eta)}{\sqrt{(v+\lambda \eta)^{2}+|D(v+\lambda \eta)|^{2}}}=\frac{D v}{\sqrt{v^{2}+|D v|^{2}}}, \\
& \lim _{\lambda \rightarrow 0} \frac{v+\lambda \eta}{\sqrt{(v+\lambda \eta)^{2}+|D(v+\lambda \eta)|^{2}}}=\frac{v}{\sqrt{v^{2}+|D v|^{2}}},
\end{aligned}
$$

for almost all $x \in \Omega$, and that

$$
\left|\frac{D(v+\lambda \eta)}{\sqrt{(v+\lambda \eta)^{2}+|D(v+\lambda \eta)|^{2}}}\right|<1 \text { and } \quad\left|\frac{v+\lambda \eta}{\sqrt{(v+\lambda \eta)^{2}+|D(v+\lambda \eta)|^{2}}}\right|<1
$$

for all $\lambda$. Also, $\lim _{\lambda \rightarrow 0} \ln (v+\lambda \eta)=\ln v$, with bounds from the height estimates on $v$ and the $L^{\infty}$ norm of $\eta$. 
By the dominated convergence theorem, we conclude

$$
\begin{aligned}
\left.\frac{\partial \mathscr{F}_{1}}{\partial \epsilon}\right|_{\epsilon=0} & =\int_{\Omega} \frac{D v}{\sqrt{v^{2}+|D v|^{2}}} \cdot D \eta d x+\int_{\Omega}\left(\frac{v}{\sqrt{v^{2}+|D v|^{2}}}+\ln v\right) \eta d x+\int_{\partial \Omega} \beta \eta d s \\
& =\lim _{\epsilon \rightarrow 0} \frac{\mathscr{g}_{1}(v+\epsilon \eta)-\mathscr{F}_{1}(v)}{\epsilon}=0,
\end{aligned}
$$

since $\mathscr{F}_{1}(v+\epsilon \eta)-\mathscr{F}_{1}(v) \geq 0$ for both positive and negative $\epsilon$.

Corollary 5.4. If $v \in H^{1,1}(\Omega)$ minimizes $\mathscr{F}_{1}$ in $\Omega$, then $u=-\ln v \in H^{1,1}(\Omega)$ is a variational solution in the sense of [Athanassenas and Finn 2006, Equation (13)], that is, satisfies the equation

$$
\int_{\Omega} \frac{D u}{\sqrt{1+|D u|^{2}}} \eta d x+\int_{\Omega} u \eta d x=\int_{\Omega} \frac{1}{\sqrt{1+|D v|^{2}}} \eta d x+\int_{\partial \Omega} \beta \eta d s,
$$

for all $\eta \in L^{\infty} \cap H^{1,1}(\Omega)$.

Theorem 5.5. Let $u \in \mathrm{BV}(\Omega)$ minimize $\mathscr{F}$, and assume $\Omega \subset \mathbb{R}^{n}$ to be open and bounded with Lipschitz boundary $\Sigma$, which satisfies an interior sphere condition. Then $u$ is analytic in $\Omega$, and there is a constant $c$ such that the gradient $|D u|$ is bounded up to the boundary of $\Omega$ :

$$
|D u| \leq c .
$$

Proof. We use the transformation $v=e^{-u}$, as defined above, and which was shown to be locally Lipschitz in $\Omega$ (Theorem 5.1). By Rademacher's theorem, $v$ is differentiable almost everywhere in $\Omega$, and as such $v \in H^{1,1}(\Omega)$, since the weak derivatives of $v$ are equal to the strong derivatives wherever they exist and also $v \in \mathrm{BV}(\Omega)$.

By Theorem 5.3, the function $v$, which minimizes $\mathscr{F}_{1}$ in the $v$ formulation of the problem, is a variational solution satisfying (5-8). By Corollary 5.4 we conclude that $u \in H^{1,1}(\Omega)$ is a variational solution in the sense of [Athanassenas and Finn 2006, Equation (13)]. In that paper the authors prove that $u$ is analytic in Lipschitz domains whose boundary $\Sigma$ satisfy an interior sphere condition, and they also obtain gradient bounds up to $\Sigma$, which concludes the proof.

\section{Acknowledgements}

This paper was begun during the first author's visit to the Max-Planck-Institut für Gravitationsphysik, Potsdam, and the second author's appointment at the Freie Universität Berlin. It was completed at the Centre for Mathematics and its Applications, Australian National University, Canberra. We thank these institutions for their support and hospitality. 


\section{References}

[Athanassenas and Finn 2006] M. Athanassenas and R. Finn, "Compressible fluids in a capillary tube”, Pacific J. Math. 224:2 (2006), 201-229. MR 2007f:76031 Zbl 1126.76049

[Bemelmans and Dierkes 1987] J. Bemelmans and U. Dierkes, "On a singular variational integral with linear growth, I. Existence and regularity of minimizers", Arch. Rational Mech. Anal. 100:1 (1987), 83-103. MR 89a:49022 Zbl 0651.49017

[Dierkes and Huisken 1990] U. Dierkes and G. Huisken, "The $n$-dimensional analogue of the catenary: existence and nonexistence”, Pacific J. Math. 141:1 (1990), 47-54. MR 90j:49031 Zbl 0652.49029

[Emmer 1973] M. Emmer, "Esistenza, unicità e regolarità nelle superfici de equilibrio nei capillari”, Ann. Univ. Ferrara Sez. VII (N.S.) 18 (1973), 79-94. MR 49 \#1281 Zbl 0275.49005

[Evans 1998] L. C. Evans, Partial differential equations, Graduate Studies in Math. 19, Amer. Math. Soc., Providence, RI, 1998. MR 99e:35001 Zbl 0902.35002

[Finn 1986] R. Finn, Equilibrium capillary surfaces, Grund. der Math. Wissenschaften 284, Springer, New York, 1986. MR 88f:49001 Zbl 0583.35002

[Finn 2001] R. Finn, “On the equations of capillarity”, J. Math. Fluid Mech. 3:2 (2001), 139-151. MR 2002c:76026 Zbl 0999.76025

[Finn 2004] R. Finn, "Capillarity problems for compressible fluids”, Mem. Differential Equations Math. Phys. 33 (2004), 47-55. MR 2005k:76116 Zbl 1101.76050

[Finn and Gerhardt 1977] R. Finn and C. Gerhardt, "The internal sphere condition and the capillary problem”, Ann. Mat. Pura Appl. (4) 112 (1977), 13-31. MR 55 \#7106 Zbl 0349.49019

[Finn and Luli 2007] R. Finn and G. K. Luli, "On the capillary problem for compressible fluids", $J$. Math. Fluid Mech. 9:1 (2007), 87-103. MR 2008i:76036 Zbl 1151.76400

[Gerhardt 1974] C. Gerhardt, "Existence and regularity of capillary surfaces", Boll. Un. Mat. Ital. (4) 10 (1974), 317-335. MR 51 \#1569 Zbl 0314.49019

[Gilbarg and Trudinger 2001] D. Gilbarg and N. S. Trudinger, Elliptic partial differential equations of second order, Springer, Berlin, 2001. Reprint of the 1998 edition. MR 2001k:35004 Zbl 1042.35002

[Giusti 1980] E. Giusti, "The pendent water drop. A direct approach", Boll. Un. Mat. Ital. A (5) 17:3 (1980), 458-465. MR 82c:49036 Zbl 0435.49004

[Giusti 1984] E. Giusti, Minimal surfaces and functions of bounded variation, Monographs in Math. 80, Birkhäuser Verlag, Basel, 1984. MR 87a:58041 Zbl 0545.49018

[Gonzalez et al. 1980] E. Gonzalez, U. Massari, and I. Tamanini, "Existence and regularity for the problem of a pendent liquid drop", Pacific J. Math. 88:2 (1980), 399-420. MR 83j:49042b Zbl 0415.49028

[Huisken 1985] G. Huisken, “Capillary surfaces over obstacles”, Pacific J. Math. 117:1 (1985), 121141. MR 87c:49047 Zbl 0561.49009

[Ladyzhenskaya and Ural'tseva 1970] O. A. Ladyzhenskaya and N. N. Ural'tseva, "Local estimates for gradients of solutions of non-uniformly elliptic and parabolic equations", Comm. Pure Appl. Math. 23 (1970), 677-703. MR 42 \#654 Zbl 0193.07202

[Serrin 1969] J. Serrin, "The problem of Dirichlet for quasilinear elliptic differential equations with many independent variables”, Philos. Trans. Roy. Soc. London Ser. A 264 (1969), 413-496. MR 43 \#7772 Zbl 0181.38003 
[Stampacchia 1966] G. Stampacchia, Èquations elliptiques du second ordre à coefficients discontinus, Séminaire Math. Sup. (Été, 1965) 16, Les Presses de l’Univ. de Montréal, 1966. MR 40 \#4603 Zbl 0151.15501

Received December 2, 2008.

MARIA ATHANASSENAS

SCHOOL OF MATHEMATICAL SCIENCES

MONASH UNIVERSITY

VIC 3800

Australia

maria.athanassenas@sci.monash.edu.au

Julie Clutterbuck

MATHEMATICAL SCIENCES Institute

Australian National UnIVERSiTy

ACT 0200

Australia

Julie.Clutterbuck@maths.anu.edu.au 